\title{
Propofol-Related Infusion Syndrome in the Peripartum Period
}

\author{
Akwugo A. Eziefule, MD ${ }^{1}$ Solafa Elshatanoufy, MD ${ }^{2}$ Mili Thakur, MD ${ }^{3,4}$ Frederico G. Rocha, MD \\ 1 Division of Maternal Fetal Medicine, Department of Obstetrics and \\ Gynecology, University of Texas Health Science Center at Houston, \\ Houston, Texas \\ 2 Division of Female Pelvic Medicine and Reproductive Surgery, \\ Department of Obstetrics and Gynecology, Henry Ford Health \\ Systems, Detroit, Michigan \\ ${ }^{3}$ Division of Reproductive Endocrinology and Infertility, Department \\ of Obstetrics and Gynecology, Wayne State University School of \\ Medicine, Detroit, Michigan \\ ${ }^{4}$ Division of Genetic and Metabolic Disorders, Department of \\ Pediatrics and Center for Molecular Medicine and Genetics, \\ Wayne State University School of Medicine, Detroit, Michigan \\ ${ }^{5}$ Department of Obstetrics and Gynecology, Hurley Medical Center, \\ Michigan State University College of Human Medicine, Flint, Michigan \\ Address for correspondence Akwugo A. Eziefule, MD, Department of \\ Obstetrics and Gynecology, University of Texas Medical School at \\ Houston, 6431 Fannin Street, MSB 3.258, Houston, TX 77030 \\ (e-mail: Akwugo.A.Eziefule@uth.tmc.edu). \\ Am J Perinatol Rep 2016;6:e368-e371.
}

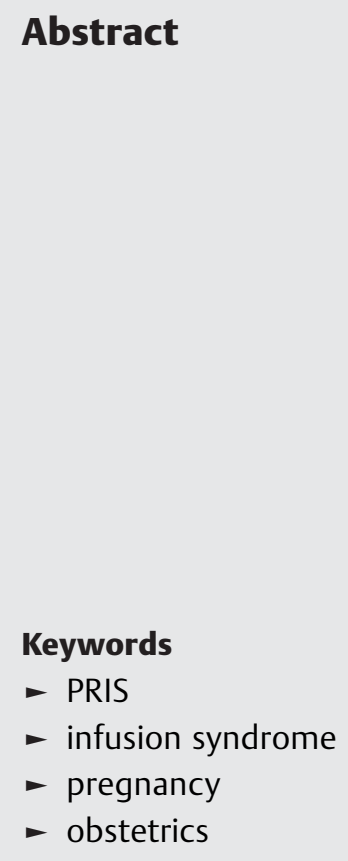

Abstract

Background Propofol is a widely known, commonly used drug. Complications can occur with the use of this drug, including propofol-related infusion syndrome (PRIS). PRIS, in the obstetric population, has not been documented; however, we report a case of a patient who developed PRIS after an emergent cesarean delivery of a preterm infant.

Case Study A 35-year-old multigravida woman presented complaining of leakage of fluid and decreased fetal movement. Her pregnancy was complicated by methadone maintenance therapy due to a history of opioid abuse. Complications after admission for prolonged monitoring and a prolonged fetal heart tone deceleration was noted with no recovery despite intrauterine resuscitation. An emergent cesarean delivery was performed using general anesthesia and endotracheal intubation after which she developed aspiration pneumonia. She was admitted to the intensive care unit and reintubation and sedation were required secondary to respiratory distress. Sedation was achieved using propofol infusion. She subsequently developed changes in her electrocardiogram, an increase of her serum creatinine, creatinine protein kinase, lipase, amylase, and triglycerides, making the diagnosis of PRIS.

Conclusion PRIS should be included in the differential diagnosis of intubated or postoperative patients in the obstetric population.

Propofol is a widely known drug used for sedation in the anesthesia and intensive care unit (ICU) population. Approved by the Food and Drug Administration in 1989, propofol has gained its popularity because of its rapid onset of action, quick recovery profile, and favorable pharmacokinetic profile. " "Propofol infusion syndrome" was first termed

by Bray in 1998 to describe a clinical state associated with propofol infusion in children. ${ }^{2,3}$ All these children exhibited a similar constellation of symptoms including metabolic acidosis, lipemic serum, and refractory bradycardia progressing to asystole. ${ }^{3}$ Also known as propofol-related infusion syndrome (PRIS), there have been many complications and adverse received

May 14, 2016

accepted after revision

August 18, 2016
DOI http://dx.doi.org/

10.1055/s-0036-1593405. ISSN 2157-6998.
Copyright $\odot 2016$ by Thieme Medical

Publishers, Inc., 333 Seventh Avenue, New York, NY 10001, USA. Tel: +1(212) 584-4662.
License terms

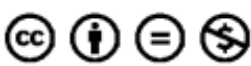


reactions associated PRIS in both adults and children, but no reports of women in the peripartum period. To our knowledge, this is the first reported case of a patient who developed PRIS after an emergent cesarean delivery of a preterm infant.

\section{Case Study}

A 35-year-old, G11 P3439, African American woman with a medical history significant for anxiety disorder, depression, and opiate abuse was well controlled on $110 \mathrm{mg}$ of methadone maintenance therapy as well as citalopram and quetiapine fumarate for 6 months before presentation. She presented to triage at $32^{4 / 7}$ weeks gestation, by her estimated delivery date, complaining of leakage of fluid per vagina and decreased fetal movement. Spontaneous rupture of the membrane was ruled out immediately with negative pooling on speculum examination, negative nitrazine test, negative ferning on microscopic evaluation of vaginal discharge, and a mean vertical pocket of $4.47 \mathrm{~cm}$ on ultrasound. The fetal nonstress test was evaluated and it was noted to have minimal variability with no accelerations and no decelerations. The patient was consequently admitted for prolonged monitoring. Approximately, 4 hours after admission there was a 7-minute fetal heart tone deceleration to $60 \mathrm{bpm}$. Despite intrauterine resuscitative measures with the administration of oxygen by mask at $10 \mathrm{~L} / \mathrm{min}$, maternal repositioning to the left decubitus position, and knee-chest position, the fetal heart rate would not recover. The decision was made to proceed with emergent cesarean delivery under general anesthesia with intubation. The patient received $200 \mathrm{mg}$ intravenous (IV) push of propofol during the induction process. She gave birth to a male infant weighing 2,040 g with Apgar score of $0,2,4$, and 7 (at 1, 5, 10, and 20 minutes, respectively).

Her immediate postoperative course was benign except for suboptimal pain control secondary to her significant narcotic tolerance. On postoperative day (POD) 3 however, the patient complained of a productive cough with yellow sputum. She remained afebrile with no clinical signs of respiratory distress. She underwent a chest X-ray that revealed a right lower lobe consolidation and a differential diagnosis of hospital-acquired pneumonia versus aspiration pneumonia, due to a recent intubation, was made. The patient was started on IV vancomycin and pipercillin-tazobactam. Subsequently, the patient complained of chest pain and was noted to be desaturating to $88 \%$ on room air. Oxygen $\left(\mathrm{O}_{2}\right)$ supplementation was administered as well as a nebulized $\beta$ agonist, but the patient continued to be tachypneic with no improvement in her oxygen saturation level. A spiral computed tomography scan was performed to rule out pulmonary embolism. An electrocardiogram (ECG) demonstrated sinus tachycardia. The patient was transferred later the same evening to the Maternal Special Care Unit (MSCU) for closer monitoring. She was controlled on $8 \mathrm{~L}$ of $\mathrm{O}_{2}$ supplementation on mask ventilation. In the MSCU, an arterial blood gas was performed demonstrating continued hypoxia and tachypnea with the following values: $\mathrm{pH}, 7.37 ; \mathrm{PO}_{2}, 64 \mathrm{~mm} \mathrm{Hg} ; \mathrm{PCO}_{2}, 45.6 \mathrm{~mm}$ $\mathrm{Hg}$; and bicarbonate, $25.9 \mathrm{mEq} / \mathrm{L}$. The patient was transferred to the medical intensive care unit secondary to worsening respiratory status and the need for intubation and sedation.
Although the patient was given IV fentanyl at $1 \mu \mathrm{g} / \mathrm{kg} / \mathrm{h}$ as well as IV midazolam at $1 \mathrm{mg} / \mathrm{h}$, the patient required extra sedation measures, perhaps secondary to her history of opiate abuse. Propofol was given as a $50 \mathrm{mg}$ intravenous bolus to aid in sedation, and a continuous IV infusion at $50 \mu \mathrm{g} / \mathrm{kg} / \mathrm{min}$ was subsequently started. With her weight recorded at $72.4 \mathrm{~kg}$, the rate of the propofol infusion was $3.6 \mathrm{mg} / \mathrm{min}$. We estimate that the patient received approximately $9,000 \mathrm{mg}$ of propofol during her intubation and sedation period of 4 days.

On POD 7, persistent acute kidney injury was noted. The patient had a rising serum creatinine level from a baseline of 0.7 to $1.4 \mathrm{mg} / \mathrm{dL}$, ECG changes, and creatinine phosphokinase (CPK) was drawn and noted to be significantly elevated at 4,585 units/L. This raised concerns for PRIS. On POD 8, 4 days after initiation of intubation, other laboratory studies were obtained including amylase, 134 units/L; lipase, 736 units/L; triglycerides, $259 \mathrm{mg} / \mathrm{dL}$; and another CPK, which was noted to be 2,626 units/L. These laboratories were all significantly elevated further supporting the diagnosis of PRIS. On POD 9, with antimicrobial therapy and corticosteroids in addition to the discontinuation of propofol, her respiratory status had dramatically improved, and the patient was successfully extubated. On POD 13, atorvastatin 20 mg daily was started for the significant hypertriglyceridemia of greater than $600 \mathrm{mg} / \mathrm{dL}$. On POD 15, her amylase was 90 units/L; lipase, 219 units/L; triglycerides, $376 \mathrm{mg} / \mathrm{dL}$; and CPK, 188 units/L. Eventually, all of her laboratories trended down toward normal and the patient was discharged home in stable condition with instructions for follow-up with all specialties of her multidisciplinary team.

\section{Discussion}

Propofol infusion syndrome is a rare, often fatal, condition of unknown etiology. Upon literature review, serious adverse reactions have occurred with patients including: metabolic acidosis, rhabdomyolysis with or without myoglobinuria, cardiac collapse, cardiac arrhythmias (most commonly bradyarrhythmias) myocardial failure, hypertriglyceridemia, renal failure, hepatomegaly, hepatic steatosis, and death all occurring after the initiation of propofol therapy. ${ }^{1-5}$ However, there is no universal definition of PRIS. Our patient did not have metabolic acidosis and only showed signs of lipemic serum (hypertriglyceridemia), acute renal failure, and abnormal cardiac function, which eventually resolved after propofol was discontinued.

The incidence of PRIS in the adult population is approximately 1.1 to $4.1 \%$ and the mortality rate is 18 to $33 \% .4$ However, determining the incidence of PRIS is complicated because many of the PRIS-associated clinical manifestations may reflect either pharmacological manifestations or critical illness (e.g., metabolic acidosis). ${ }^{6}$ In addition to the confusion regarding the true incidence of PRIS, there remains no consensus on the management of PRIS other than early recognition and discontinuation of propofol. ${ }^{4}$ Within 5 days of the patient receiving the first propofol bolus, it was noted that she developed sinus tachycardia with a productive cough. The differential diagnosis was nosocomial versus 
aspiration pneumonia. Moreover, after the patient was intubated for the second time, requiring a continuous infusion of propofol, the patient developed acute kidney injury, however, the cause was attributed to use of contrast with the computed tomography scan and furosemide.

Propofol infusion syndrome is defined clinically, and although definitions vary, metabolic acidosis is a common factor. ${ }^{7}$ In addition to clinical manifestations, abnormal laboratory values include, lactate, creatine kinase, triglycerides, and ST-segment elevation in right precordial leads. ${ }^{6}$ On POD 5 the patient was intubated for a second time due to worsening symptoms and a continuous infusion of propofol was started thereafter. By POD 11 the medical team noted her lipase was twice the normal limit as well as an increase in CPK and triglycerides levels. During this time, the patient continued to have ECG changes including sinus bradycardia with prolonged QT interval, anterior ischemia, and abnormal T-wave abnormality. After discontinuation and by the time of discharge, all of the patients ECG abnormalities resolved and she was scheduled to follow-up with cardiology. The most commonly reported laboratory and clinical findings are metabolic acidosis (88.2\%), rhabdomyolysis (64.7\%), cardiac arrhythmias (70.5\%), and hypotension (52.9\%). Less commonly described presentation included renal failure (47\%), hyperkalemia (44.1\%), and hyperlipidemia (20.5\%). ${ }^{1}$

It has been suggested that a minimum dose of propofol is required to develop this syndrome. Many studies state that in cases of PRIS, patients who were on $>4 \mathrm{mg} / \mathrm{kg} / \mathrm{h}$ for $>48$ hours, developed similar symptoms. ${ }^{2-4,6}$ The recommended induction dose of propofol given as an infusion in adults requiring sedation in an ICU is $0.3 \mathrm{mg} / \mathrm{kg} / \mathrm{h}$, which may be increased by 0.3 to $0.6 \mathrm{mg} / \mathrm{kg} / \mathrm{h}$ until the desired level of sedation is achieved. ${ }^{1}$ Our patient was noted to have received over 9,000 mg of propofol during her hospital stay, well over the recommended limit that can cause PRIS. We suspect this was due to her profound opioid tolerance that caused her to require high doses of medication to induce sedation.

In 1996, Merinella was the first author to suggest that propofol reaction should be included in the differential diagnosis of metabolic acidosis developing in adult patients during long-term sedation with propofol. ${ }^{4,5}$ PRIS remains a complex and multifaceted clinical syndrome, and the overwhelming majority of patients diagnosed with PRIS have significant preexisting and overlapping comorbidities. ${ }^{4}$ In our case, the patient first showed signs of hypoxia, likely due to the developing pneumonia that was diagnosed and lead to her intubation. Usual sedation practices include midazolam and fentanyl as in our patient, however, propofol was added because the patient remained combative and attempted self-extubation several times. We believe that her history of opiate abuse and a high dose of methadone could have contributed to this.

The mechanism responsible for PRIS remains controversial. The leading hypothesis (-Fig. $\mathbf{1}$ ) is that propofol causes metabolic derangements by affecting $\beta$-oxidation of free fatty acids (FFAs). ${ }^{4}$ Cardiac muscle uses FFAs in preference to

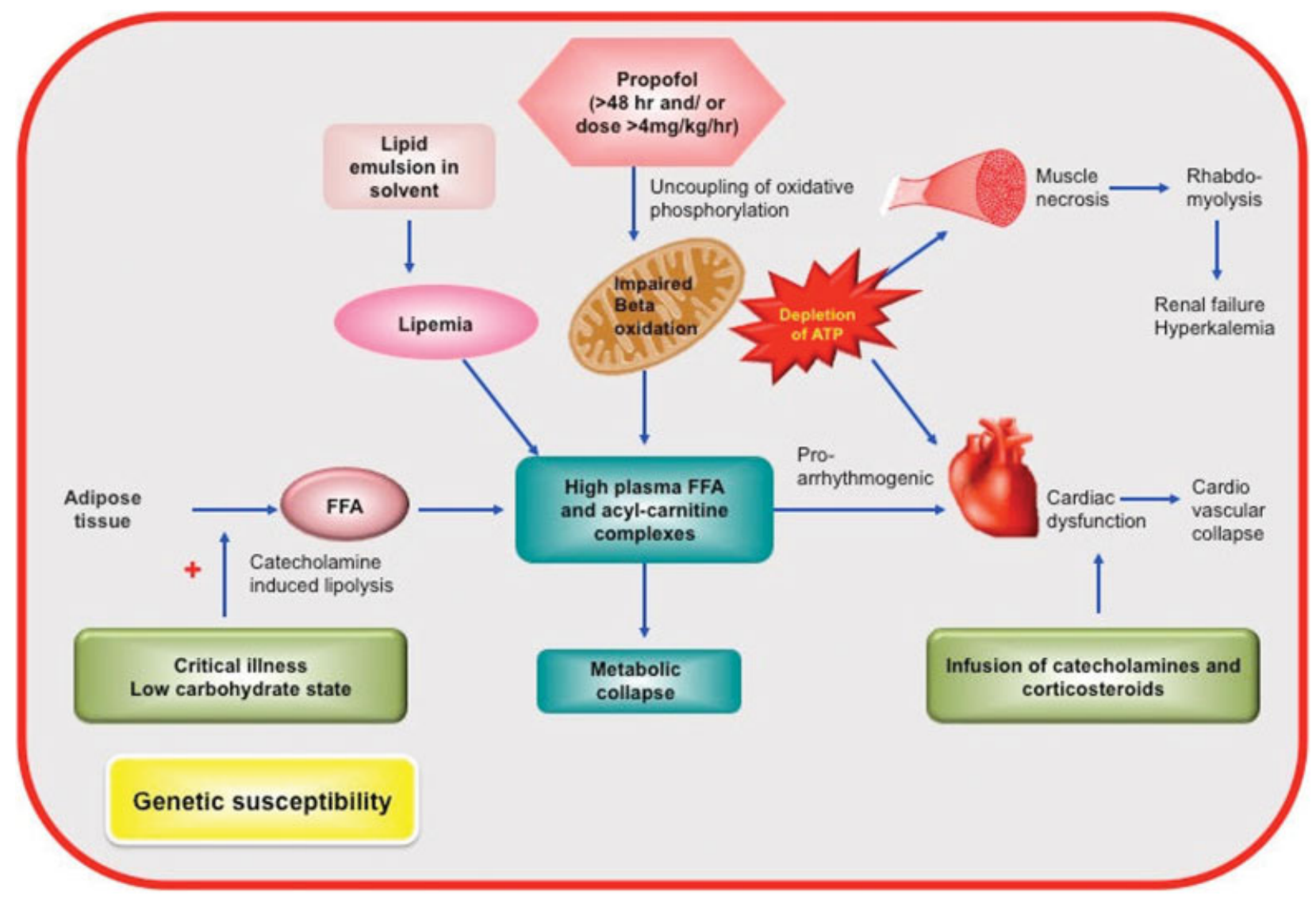

Fig. 1 Pathophysiology of propofol-related infusion syndrome. ATP, adenosine triphosphate; FFA, free fatty acids. 
glucose. FFAs are also the main source of energy for skeletal muscles during sustained exercise. During critical illness and in states of carbohydrate deprivation, catecholamines induce lipolysis to release FFAs from the adipose tissue, so these can be used to derive energy and spare glucose for the brain. The FFAs undergo $\beta$-oxidation in the mitochondria to generate adenosine triphosphates (ATPs) by passing electrons to the electron transport chain. Acetyl-CoA is also released which is oxidized in the Krebs cycle, or is used to generate ketone bodies in the liver. Propofol inhibits the electron transport chain causing uncoupling of oxidative phosphorylation. This decreases the mitochondrial transmembrane potential and thus affects transportation of FFAs into the mitochondria. Loss of ATP production leads to widespread cell death resulting in hepatic dysfunction, multiorgan failure, and metabolic collapse. Lactic acidemia results from inhibitory effects of acylcarnitine metabolites on various steps of pyruvate metabolism, contributing to metabolic acidosis. Due to an imbalance in energy demand and supply, there is cardiac dysfunction ultimately leading to cardiovascular collapse. Accumulating metabolites are also proarrhythmogenic, and thus, the electrocardiographic changes. Skeletal muscle necrosis leads to rhabdomyolysis. Myoglobin release leads to acute renal failure and hyperkalemia. Infusion of catecholamines and corticosteroids in the setting of critical illness also contribute to myopathy. In patients with PRIS, lipemia experienced results from the lipid emulsion in the solvent and from accumulating FFAs. Despite the widespread use of propofol, PRIS has been rarely reported. Genetic susceptibility to develop this condition has therefore been postulated. However, the genetic predisposition has not been identified. Thus, we suggest that a toxic effect on mitochondria occurring only in genetically susceptible individuals could account for the sporadic nature of propofol-related metabolic acidosis., ${ }^{2,7}$ In some cases, PRIS is associated with the genetically proven mitochondrial disease. ${ }^{8,9}$ It is unknown whether the patient had this underlying comorbidity. Maintaining adequate carbohydrate intake in critically ill patients may prevent the switch to fat metabolism and thus prevent the onset of PRIS. ${ }^{4}$

Risk factors for PRIS include critical illness, propofol dosage $>4 \mathrm{mg} / \mathrm{kg} / \mathrm{h}$, duration of therapy $>48$ hours, exogenous catecholamines and corticosteroids, poor carbohydrate intake, severe head injury, airway infection, young age, large total cumulative dose, low carbohydrate intake/high fat intake, or inborn errors of fatty acid oxidation. ${ }^{3,6}$ Our patient had many risk factors that contributed to her clinical outcome. She received propofol over a 5-day period, she had an airway infection with a diagnosis of aspiration pneumonia with possible bacterial infection, her calculated total dose of propofol was approximately $9,000 \mathrm{mg}$, and she did not have any carbohydrate intake during her ICU admission. The increased metabolic demand in the peripartum period, the relative tissue hypoxia secondary to the postoperative state and sepsis may have impacted the development of this condition in our patient.

In conclusion, propofol should not be used for sedation for more than 3 days if possible. During propofol infusions, clinicians should monitor arterial blood gases, serum triglycerides, creatine kinase, all electrolytes (particularly potassium), serum lactate levels, liver function tests, blood urea nitrogen, and creatinine. ${ }^{4}$ Prevention of PRIS should include the use of the lowest dose of propofol with the shortest duration, minimal lipid load (concentrating propofol drip and adjusting parenteral nutrition), and provide an adequate amount of carbohydrate. A propofol infusion should be stopped at the earliest sign of abnormal laboratory results or ECG changes. ${ }^{3,6}$

Funding

None.

Conflict of Interest

None.

\section{References}

1 Orsini J, Nadkarni A, Chen J, Cohen N. Propofol infusion syndrome: case report and literature review. Am J Health Syst Pharm 2009; 66(10):908-915

2 Bray RJ. Propofol infusion syndrome in children. Paediatr Anaesth 1998;8(6):491-499

3 Wong JM. Propofol infusion syndrome. Am J Ther 2010;17(5): 487-491

4 Diaz JH, Prabhakar A, Urman RD, Kaye AD. Propofol infusion syndrome: a retrospective analysis at a level 1 trauma center. Crit Care Res Pract 2014;201:346968

5 Roberts RJ, Barletta JF, Fong JJ, et al. Incidence of propofol-related infusion syndrome in critically ill adults: a prospective, multicenter study. Crit Care 2009;13(5):R169

6 Imam TH. Propofol-related infusion syndrome: role of propofol in medical complications of sedated critical care patients. Perm J 2013;17(2):85-87

7 Chukwuemeka A, Ko R, Ralph-Edwards A. Short-term low-dose propofol anaesthesia associated with severe metabolic acidosis. Anaesth Intensive Care 2006;34(5):651-655

8 Savard M, Dupré N, Turgeon AF, Desbiens R, Langevin S, Brunet D. Propofol-related infusion syndrome heralding a mitochondrial disease: case report. Neurology 2013;81(8):770-771

9 Vanlander AV, Jorens PG, Smet J, et al. Inborn oxidative phosphorylation defect as risk factor for propofol infusion syndrome. Acta Anaesthesiol Scand 2012;56(4):520-525 